\title{
Integrated Clustering and Routing Strategies for Large Scale Sensor Networks
}

\author{
Ataul Bari, Arunita Jaekel, and Subir Bandyopadhyay \\ School of Computer Science, University of Windsor \\ 401 Sunset Ave. Windsor, ON N9B 3P4, Canada \\ \{bari1, arunita, subir\}@uwindsor.ca
}

\begin{abstract}
In two-tiered sensor networks using relay nodes, sensor nodes are arranged in clusters and the higher-powered relay nodes can be used as cluster heads. The lifetime of the networks is determined primarily by the lifetime of the relay nodes. Clustering techniques and routing schemes play a crucial role in determining the useful network lifetime. Traditionally, the clustering and the routing problems, for these networks, have been considered independently and solved separately. In this paper, we present a new integer linear program (ILP) formulation that jointly optimizes both clustering and routing to maximize the lifetime of such networks. We show that our integrated approach can lead to significant improvements over techniques that consider clustering and routing separately, particularly for the non-flow-splitting (single-path) routing model. We also propose a heuristic, based on LP-relaxation of the routing variables, which can be used for larger networks.
\end{abstract}

\section{Introduction}

A wireless sensor network (WSN) is a network of low-powered, multi-functional sensor nodes, each consisting, at a minimum, of a sensing circuit, a digital signal processor, and radio links [1], [2, 3]. It is extremely important to design routing protocols and algorithms that are energy efficient, so that the overall lifetime of the network can be extended as much as possible. In a two-tier hierarchical architecture, the network is organized as a number of clusters where each sensor node belongs to only one cluster. Some nodes are treated as cluster heads and have additional responsibilities (e.g. data gathering, data aggregation and routing) compared to the remaining nodes. Recently, relay nodes, acting as cluster heads, have been proposed in two-tier sensor networks [2, 4], [5, 6], 7], 8], 9] for energy-efficient data gathering, load-balancing and fault tolerance [2], 5], 7].

In relay-based networks, each relay node collects data from the sensor nodes in its cluster and forwards this data to the base station (or sink) using either single-hop or multi-hop communication model. The multi-hop data transmission model (MHDTM), 7], 8, 9, 10] is particularly suitable for larger networks and is the model used in this paper.

A number of routing schemes for two-tiered networks have been proposed in the literature [2, 4], [5], [7, 8], 10. Most of these adopt the flow-splitting (also 
called multi-path routing) model. In contrast, in a single-path routing model, a node is not allowed to split the traffic, and forwards all its data to a single neighbor. This model avoids many limitations of the flow splitting model [7.

An important factor affecting the lifetime of a two-tier network is the clustering scheme used to assign sensor nodes to the appropriate clusters [6], [1], particularly for single-path routing. Previous approaches to clustering and routing have considered the two problems independently. Typically, the assignment of sensor nodes to clusters is done first, and then a routing scheme to maximize the network lifetime is calculated.

In this paper, we present a new integer linear program (ILP) formulation that jointly optimizes both clustering and routing to maximize the lifetime of the upper-tier relay node network. We have assumed a network model where a) the roles of the sensor nodes and the relay nodes are not interchangeable, b) the relay nodes do not perform sensing tasks and are provisioned with higher energy, c) the relay nodes can transmit over larger distances, compared to regular sensor nodes, d) each sensor node, is located close enough to some relay node so that the sensor node can transmit directly to the relay node, e) sensor nodes only communicate to their respective cluster heads and do not take part in the routing, and $\mathrm{f}$ ) both the sensor nodes and the relay nodes communicate through an ideal shared medium where communication between nodes is handled by appropriate MAC protocols (as in [2, [5]).

We focus on the non-flow-splitting (single-path) routing model and show that our integrated approach can lead to significant improvements over techniques that consider clustering and routing separately. To the best of our knowledge, this is the first technique that combines the clustering and the routing problem to maximize the network lifetime.

We assume that the clustering and the routing scheme is computed at the base station (or at some centralized entity where such computation may be carried out). There are two possible scenarios for determining the positions of the relay nodes and the sensor nodes as follows:

Case i) We place the sensor and the relay nodes at predetermined locations. Before the deployment of the network, we can compute the clustering and the routing decisions at some centralized location. The sensor nodes and the relay nodes may be pre-configured with this information.

Case ii) We can find the locations of the nodes using a GPS system. GPS equipped nodes has been widely proposed in the literature [1], 2], [5], 6]. The GPS system needs to be operated for a very short period of time, to know the locations of the sensor and the relay nodes. We also assume that the nodes are stationary after deployment. We can then compute, at some central location, the clustering and the routing decisions and broadcast the result to the entire network. Since the communication from each sensor or relay node as well as the communication broadcast from the base station to the sensor and relay nodes will be a single, small packet, the energy 
dissipated for sending/receiving these two packets is insignificant, compared to the subsequent transmissions, and will not have any substantial impact on the lifetime of the network.

In this paper we have

i) presented an integrated ILP for optimal clustering and single-path routing to maximize network lifetime, giving a significant increase in network lifetime compared to solving the two problems separately.

ii) proposed a LP-relaxation of the routing variables so that our approach can be used for multi-path routing as well.

iii) extended the above ILP to develop a new heuristic for single-path routing capable of handling large networks, and have shown, through simulations, that the network lifetimes that may be achieved by the heuristic solutions is close to the theoretical upper bound.

\section{Review}

\subsection{Load Balanced Clustering}

Many researchers have investigated clustering of nodes in a wireless network [2], [3], [5]. In 2], the problem of forming clusters around a few high-energy gateway nodes has been investigated. In Fig. 1, the sensor nodes in the shaded region can be assigned to any one of clusters $\mathrm{A}, \mathrm{B}$ or $\mathrm{C}$. The routing scheme and/or the energy dissipation of relay nodes A, B and C, may favor one assignment over the others. A load balanced clustering algorithm assigns each sensor node to an appropriate cluster to maximize the lifetime of the network.

In 9, a minimum number relay nodes have been used as cluster heads to cover all sensor nodes. However, 9] does not address the issue of clustering after the placement of the relay nodes. In [2, the "cardinality" of a cluster (the number of sensor nodes associated with the cluster) is used in a heuristic to minimize the variance of the cardinality of each cluster in the network. In [6], it is demonstrated that suitable clustering techniques can be used to increase the lifetime of the network.

\subsection{Routing in Sensor Networks Using Relay Nodes}

The problem of routing in wireless sensor networks, under the "flow-splitting" model, has been extensively covered in the literature. In 8, Hou et al. have attempted to maximize the lifetime of a sensor network by provisioning relay and sensor nodes with additional energy using a mixed-integer non-linear program and have proposed a heuristic. In [10, the authors have formulated the lifetime optimization problem, under the flow-splitting model. In [12, Falck et al. have addressed the issue of balanced data gathering in sensor networks and have proposed a LP formulation that enforces some balancing constraints in the data gathering schedule. In 2, Gupta and Younis have focused on load balanced 


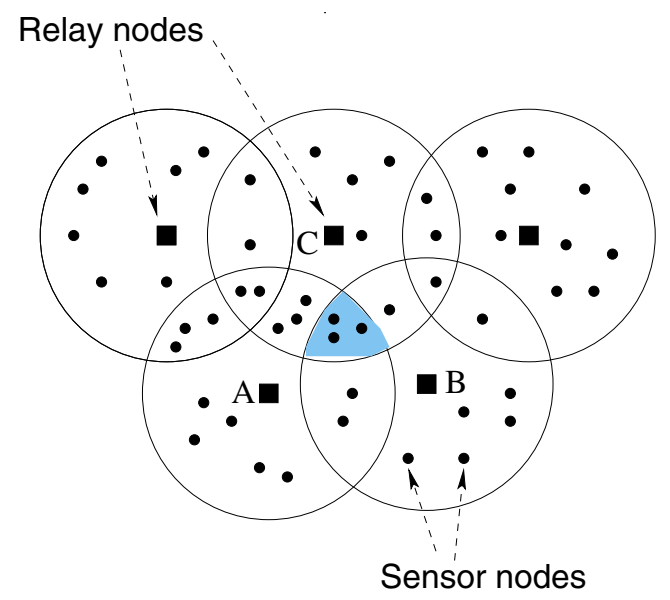

Fig. 1. Sensor nodes in overlapping coverage area

clustering and have proposed a heuristic solution for the optimization problem. Routing without flow splitting (i.e., single-path routing) has been studied in [7], [13], and [14]. In [7], the authors have presented a transformation algorithm to convert a multiple outgoing flow routing model to a single outgoing flow routing model. In [14], the authors have investigated the problem of maximizing network lifetime by appropriately placing nodes which are not energy constrained (e.g., connected to a wall outlet). In [13], the authors propose a formulation for constructing minimum-energy data-aggregation trees, for a flat architecture.

\section{ILP Formulations for Optimal Clustering and Routing}

\subsection{Network Model}

In this paper, we have assumed that the communication energy dissipation is based on the first order radio model [3], where the energy required to transmit (receive) $b$ bits, at a distance $d$, is given by $E_{T_{x}}(b, d)=\alpha_{1} b+\beta b d^{m}\left(E_{R_{x}}(b)=\right.$ $\left.\alpha_{2} b\right)$, where $\alpha_{1}\left(\alpha_{2}\right)$ is the energy coefficient for transmitter (receiver), $\beta$ is the energy coefficient for the transmit amplifier and $q$ is the path loss exponent.

For our network model, we consider a two-tiered wireless sensor network with $n$ sensor nodes, $m$ relay nodes and one base station. Each sensor node belongs to only one cluster and each relay node acts as the cluster head of exactly one cluster. We assign each node a unique label as follows:

i) for each sensor node, a label $i, 1 \leq i \leq n$,

ii) for each relay node, a label $j, n<j \leq n+m$, and

iii) for the base station, a label $n+m+1$.

Let $\mathcal{S}$ be the set of all sensor nodes. Each sensor node belongs to only one cluster and each relay node acts as the cluster head of exactly one cluster. In 
other words, if $\mathcal{S}^{j}, n+1 \leq j \leq m+n$, is the set of sensor nodes belonging to the $j^{t h}$ cluster, then $\mathcal{S}=\mathcal{S}^{n+1} \cup \mathcal{S}^{n+2} \cup \ldots \cup \mathcal{S}^{m+n}$ and $\mathcal{S}^{j} \cap \mathcal{S}^{k}=\emptyset, \forall j \neq k, n+1 \leq$ $j, k \leq m+n$. The set $\mathcal{S}^{j}$ will constitute the cluster with the relay node having label $j$ as the cluster head.

As mentioned in the introduction, we assume that the locations of all the sensor nodes and the relay nodes are known (or can be determined), and the average amount of data generated by each sensor node is also known a priori. The data rate for all sensor nodes need not be uniform, but can vary from node to node. We also assume that the placement strategy, applied during the deployment phase of the network, ensures proper coverage of each sensor node and the connectivity of the relay node network.

In our model, data gathering is proactive, i.e., data are collected and forwarded to the base station periodically, following a predefined schedule. We refer to each period of data gathering as a round [10. In each round of data gathering, each relay node gathers the data it receives from its own cluster and transmits that data, either directly to the base station (single hop model) or forwards the data towards the base station using a multi-hop path (multi-hop model). In the case of multi-hop routing, in addition to the data generated by its own cluster, each relay node also relays any data it receives from neighboring relay nodes. We will measure the lifetime of the network by the number of rounds the network operates from the start, until a relay node depletes its energy completely and ceases to function.

\subsection{Notation Used}

In our formulation we are given the following data as input:

- $\alpha_{1}\left(\alpha_{2}\right)$ : Energy coefficient for transmitter (receiver).

- $\beta$ : Energy coefficient for amplifier.

- $q$ : Path loss exponent.

- $b_{i}$ : Number of bits generated by sensor node $i$ per round.

- $n(m)$ : Total number of sensor (relay) nodes, with each sensor (relay) node having a unique index lying between 1 and $n(n+1$ and $n+m)$.

- $n+m+1$ : Index of the base station.

- $C$ : A large constant, greater than $\sum b_{i}$, the total number of bits received by the base station in a round.

- $r_{\max }\left(d_{\max }\right)$ : Transmission range of each sensor (relay) node.

- $d_{i, j}$ : Euclidean distance from node $i$ to node $j$.

We also define the following variables:

- $X_{i, j}$ : Binary variable defined as follows:

$$
X_{i, j}=\left\{\begin{array}{l}
1 \text { if sensor node } i \text { belongs to the cluster } \\
\quad \text { of relay node } j \\
\forall i, j: 1 \leq i \leq n, n+1 \leq j \leq n+m \\
0 \text { otherwise }
\end{array}\right.
$$


- $Y_{j, k}$ : Binary variable defined as follows: $Y_{j, k}=\left\{\begin{array}{l}1 \text { if relay node } j \text { transmits to relay node } k, \\ 0 \text { otherwise. }\end{array}\right.$

- $B_{j}$ : Total number of bits generated by the sensor nodes belonging to cluster $j$ in one round.

- $f_{j, k}$ : Number of bits sent by relay node $j$ to relay node $k$ in one round.

- $R_{j}$ : Number of bits received by relay node $j$ from other relay nodes in one round.

- $T_{j}$ : Number of bits transmitted by relay node $j$ in one round.

- $F_{\text {max }}$ : The total energy spent per round by the relay node which is being depleted at the fastest rate.

\subsection{ILP Formulation for Non Flow-Splitting Model (ILP-NFS)}

Given the network as described in Section 3.1, the objective of this formulation is to maximize the lifetime of the network by finding an optimal clustering and routing scheme.

\section{Minimize $F_{\max }$}

Subject to:

a) A sensor node $i$ can transmit to a cluster-head $j$, only if the distance between $i$ and $j$ is less than the range $r_{\max }$ of the sensor node.

$$
\begin{array}{ll}
X_{i, j} \cdot d_{i, j} \leq r_{\max }, \quad & \forall i, 1 \leq i \leq n, \\
& \forall j, n<j \leq n+m
\end{array}
$$

b) A sensor node must belong to exactly one cluster.

$$
\sum_{j=n+1}^{n+m} X_{i, j}=1, \quad \forall i, 1 \leq i \leq n
$$

c) Compute the total number of bits $B_{j}$ received at relay node $j$ from its own cluster in one round of data gathering.

$$
\sum_{i=1}^{n} b_{i} \cdot X_{i, j}=B_{j}, \quad \forall j, n<j \leq n+m
$$

d) Relay node $j$ can only transmit to one relay node or to the base station (non-flow-splitting constraint).

$$
\sum_{k=n+1}^{n+m+1} Y_{j, k}=1, \quad \forall j, n<j \leq n+m
$$


e) Compute the total number of bits $T_{j}$ transmitted by relay node $j$ in one round of data gathering.

$$
T_{j}=\sum_{k=n+1}^{n+m+1} f_{j, k}, \quad \forall j, n<j \leq n+m
$$

f) Compute the total number of bits $R_{j}$ received at node $j$ (a relay node or the base station) from other relay nodes in one round of data gathering.

$$
R_{j}=\sum_{k=n+1}^{n+m} f_{k, j}, \quad \forall j, n<j \leq n+m+1
$$

g) Ensure that relay node $j$ is transmitting to relay node $k$, only if relay node $k$ is the next node in the multi-hop path from $j$ to the base station (or $k$ is the base station).

$$
\begin{aligned}
f_{j, k} \leq C \cdot Y_{j, k}, \quad \forall j, k, n & <j \leq n+m, \\
n & <k \leq n+m+1
\end{aligned}
$$

h) A relay node transmits data gathered from its own cluster as well as any data it receives from other relay node(s).

$$
T_{j}=B_{j}+R_{j}, \quad \forall j, n<j \leq n+m
$$

i) A source relay node can transmit to a destination relay node (or base station) if the destination node is within the transmission range of the source node.

$$
\begin{aligned}
Y_{j, k} \cdot d_{j, k} \leq d_{\max }, \forall j, k, \quad & n<j \leq n+m, \\
& n<k \leq n+m+1
\end{aligned}
$$

j) Compute the total energy dissipated by each relay node $j$ in one round of data gathering.

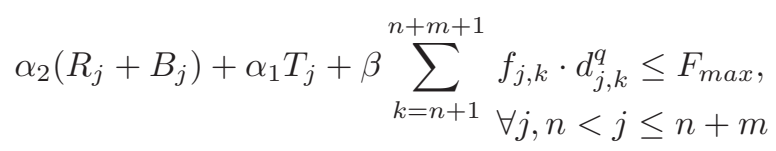

In evaluating ILP-NFS, we have computed the lifetime as the number of rounds until one relay node becomes completely depleted. Although ILP-NFS minimizes the maximum load in any relay node in a round, it is possible that, after the first relay node is depleted, other relay nodes might still have some residual power. We could have used the surviving relay nodes to further extend the lifetime of the network. The rescheduling strategy we have proposed in [4] may be used, where the routes are recomputed at periodic interval 1 , taking into consideration the available residual energy of each relay node. We have shown in 4. that it is possible to increase significantly the lifetime of the network by this technique. We may apply the same technique to this formulation as well. We omit the details since the technique is essentially the same.

${ }^{1}$ Clustering was not considered in that paper. 


\section{LP-Relaxation Based Heuristic}

The ILP formulation presented in the previous section guarantees an optimal solution that maximizes the lifetime of the upper-tier relay node network. However, this formulation becomes computationally intractable for larger networks. In this section, we propose a heuristic approach based on a LP-relaxation of the routing variables $Y_{j, k}$. In this approach, we first solve the ILP (ILP-FS) for the combined clustering and routing problem under the flow-splitting model. This means that we allow the traffic to be split among different nodes, and no longer require the integer variables $Y_{j, k}$. The solution obtained for the flow-splitting model is then used to guide the ILP for the non-flow-splitting model. The idea is that, we define a small set $\mathcal{P}^{j}$ of "promising" relay nodes, for node $j$, for all $j, n<j \leq n+m$. The nodes in $\mathcal{P}^{j}$ are selected, based on the nodes that $j$ transmits to, in the solution generated by ILP-FS. When looking for the next node, in the path from relay node $j$ to the base station, the new formulation only considers the nodes in $\mathcal{P}^{j}$.

\subsection{ILP for Flow-Splitting Model (ILP-FS)}

The formulation for the flow-splitting model is similar to ILP-NFS presented in the previous section, with the following modifications.

1. The variables $Y_{j, k}$ are eliminated. Since, we are allowed to arbitrarily split the traffic, the integer routing variables $Y_{j, k}$ are no longer needed and traffic flows can be determined by the continuous flow variables $f_{j, k}$.

2. Since a relay node can transmit to any number of other nodes, constraint (5) is no longer needed, and is removed.

3. Since there can be non-zero flow to more than one node, constraint (8) is not needed, and is removed.

4. Constraint (10) is modified as follows:

$$
f_{j, k}=0, \quad \forall j, k, \ni d_{j, k}>d_{\max }
$$

All other variables and constraints, as well as the objective function are identical to ILP-NFS.

\subsection{Non Flow Splitting-Heuristic (NFS-H)}

In the heuristic, we find the routing scheme in three steps. The first step in the heuristic is to run ILP-FS to identify the set, $\mathcal{P}^{j}$, of promising relay nodes for the $j^{\text {th }}$ relay node. In the second step, we set $Y_{j, k}=0$, for all relay nodes not in $\mathcal{P}^{j}$. Finally, we run ILP-NFS to generate a solution for combined clustering and routing, under non-flow splitting model. By setting $Y_{j, k}=0$, in step 2, for all relay nodes not in $\mathcal{P}^{j}$, ILP-NFS is forced to select one of the nodes in $\mathcal{P}^{j}$ as the next node in the path from node $j$ to the base station. By extensive simulation, we have observed that the size of $\mathcal{P}^{j}$, for each node $j$, is typically from 1 to 4 . This considerably reduces the complexity for ILP-NFS and we find that ILP-NFS quickly converges to a solution. 


\section{$5 \quad$ Experimental Results}

\subsection{Experimental Setup}

We have carried out a number of experiments to test the effectiveness of our formulations, with different sensor and relay node distributions. We have measured the achieved lifetime of a network by the number of rounds until the first relay node runs out of battery power. The ILP formulations were solved using CPLEX version 9.1 [15. In the first set of experiments, we consider networks with up to 15 relay nodes, and sensor nodes randomly distributed over the region. We varied the number of sensor nodes from 100 to 500 nodes. To evaluate the performance of our approach, we compared the achieved lifetime with standard multi-hop routing schemes such as the minimum hop (MH) routing and the minimum transmission energy (MTE) routing. We also considered an optimal single-path routing scheme (ILP-R) 4, which maximizes the network lifetime for a specified clustering strategy. For each routing scheme, we experimented with a number of standard clustering techniques [11] such as:

i. Greedy Clustering (GC): Each relay node greedily selects all sensor nodes which can communicate with it, and has not yet been assigned to a cluster.

ii. Least Distance Clustering (LDC): Each sensor node transmits to the closest relay node.

iii. Minimum Variance Clustering (MVC): The clustering algorithm attempts to distribute sensor nodes uniformly over the relay nodes, such that the variation in cluster size is minimized.

In the following sections, we have shown the results corresponding to the MVC clustering algorithm, which is the best performing algorithm as reported in [6. The improvements obtained with respect to GC and LDC are comparable to MVC, or even higher. The first set of experiments was used to calibrate the performance of the heuristic with respect to the optimal. In the second set of experiments, we considered much larger networks with up to 44 relay nodes and 5000 sensor nodes. For such networks, it was not possible to obtain optimal solutions using the ILP for the non-flow-splitting model. So, we compared our heuristic with MTE and MH routing.

We have assumed that the communication energy dissipation is based on the first order radio model, described in Section 3.1 The values for the constants are taken as $\alpha_{1}=\alpha_{2}=50 \mathrm{~nJ} / \mathrm{bit}, \beta=100 \mathrm{pJ} / \mathrm{bit} / \mathrm{m}^{2}$ and the path-loss exponent, $q=2$, which are similar to 3 . The range of each sensor (relay) node is set to $40 \mathrm{~m}(200 \mathrm{~m})$ and the initial energy of each relay node is set to $5 J$, as in $[9]$.

\subsection{Performance Evaluation for Moderate Sized Networks}

In this section, we present the results for a $160 \mathrm{~m} \times 160 \mathrm{~m}$ area network with 12 relay nodes. Figure 2 shows the network lifetime obtained by using our integrated approach versus the MH, MTE, and ILP-R routing strategies combined with MVC clustering scheme, for the non-flow-splitting model. For each data set, we 
represent the lifetimes achieved by the different strategies in the following order: minimum-hop routing $(\mathrm{MH})$, minimum transmission energy routing (MTE), optimal routing performed separately after clustering (ILP-R), LP-relaxation based heuristic for combined clustering and routing (NFS-H), and optimal solution for combined clustering and routing (ILP-NFS).

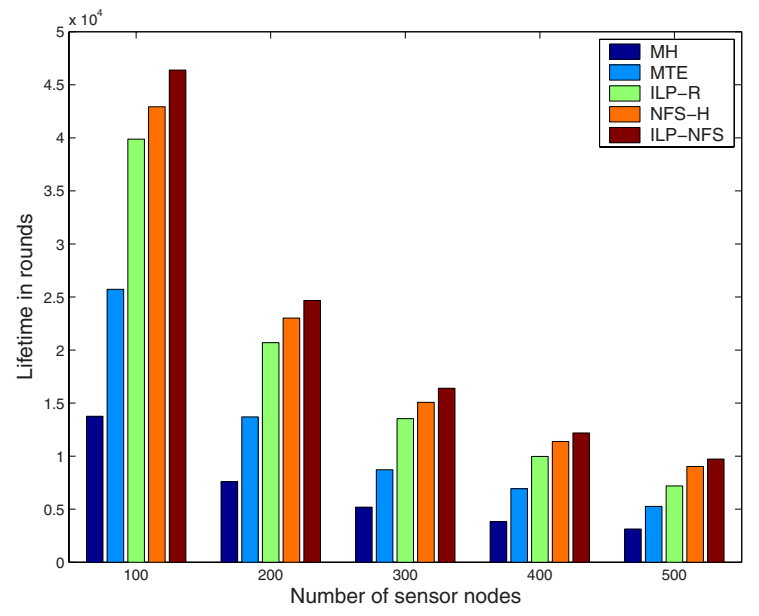

Fig. 2. Comparison of achieved lifetimes with MVC scheme

The data clearly indicate that an integrated approach performs significantly better than traditional routing schemes. The average achieved lifetime obtained by ILP-NFS increased by over 300\% (over 200\%) compared to traditional routing schemes such as MH (MTE). Even when compared to the optimal routing generated by ILP-R, ILP-NFS produced an average improvement of $23 \%$. The results also show that the performance of our heuristic (NFS-H) is quite close to the optimal (within 10\% - 15\%) in all cases.

\subsection{Performance Evaluation for Large Scale Networks}

In the previous sections we compared our heuristic (NFS-H), with the optimal solution and have shown that its performance is close to optimal for small networks. For large networks, it was not possible to generate optimal solutions for the non-flow-splitting model, using ILP-NFS. The optimal routing formulation (ILP-R) in [4] was also unable to generate solutions for larger networks. Therefore, in this section, we compare the performance of our heuristic with traditional $\mathrm{MH}$ and MTE routing schemes. We also report the achieved lifetime obtained using ILP-FS. This provides an upper bound on the maximum achievable lifetime for each experimental run. 


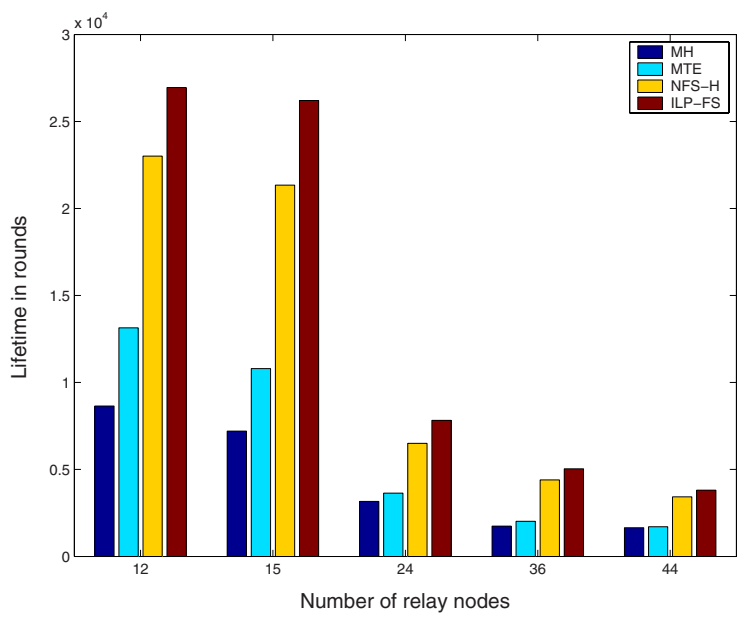

Fig. 3. Comparison of achieved lifetimes for moderate to large networks

Figure 3 shows how the achieved lifetime varies with the size of the network. For a specific size of relay node network, we selected the number of sensor nodes so that the average cluster size remains the same for all networks. The sensing area varied from $160 \mathrm{~m} \times 160 \mathrm{~m}$ for a network with 12 relay nodes to $400 \mathrm{~m}$ $\times 280 \mathrm{~m}$ for a network with 44 relay nodes. As before, Figure 3 confirms that the combined approach clearly performs better than both $\mathrm{MH}$ and MTE for large networks. The average improvement of our combined approach is $300 \%$ and $200 \%$ over MH and MTE respectively. The overall lifetime decreases with network size, since the sensing area as well as the total amount of data to be transmitted increases, which in turn increases the load on critical nodes close to the base station.

\section{Conclusion}

In this paper we have proposed an integrated approach that jointly optimizes clustering and routing in large scale two-tier sensor networks. We have presented an ILP formulation that maximizes the lifetime of the upper-tier relay node network, as well as a LP-relaxation based heuristic that can be used for large networks with thousands of nodes. We have calibrated the performance of the heuristic, by comparing with optimal solutions for smaller networks. We have demonstrated that our combined approach significantly increases network lifetime for non-flow-splitting (single-path) routing. Our proposed heuristic clearly outperforms traditional routing schemes such as minimum-hop routing and minimum-transmission-energy routing, for large scale networks. 


\section{Acknowledgment}

The work of A. Jaekel and S. Bandyopadhyay have been supported by research grants from the Natural Sciences and Engineering Research Council of Canada (NSERC).

\section{References}

1. I.F. Akyildiz, W. Su, Y. Sankarasubramaniam, and E. Cayirci. Wireless sensor networks: a survey. Computer Networks, vol. 38, pp. 393-422, 2002.

2. G. Gupta and M. Younis. Load-balanced clustering of wireless sensor networks. In IEEE Intl. Conf. on Comm., vol. 3, pp. 1848-1852, 2003.

3. W. Heinzelman, A. Chandrakasan, and H. Balakrishnan. Energy effcient communication protocol for wireless micro-sensor networks. In Proc. of the 33rd HICSS, pp. 3005-3014, 2000.

4. A. Bari, A. Jaekel and S. Bandyopadhyay. Maximizing the Lifetime of Two-Tiered Sensor Networks. In IEEE EIT, pp. 222-226, 2006.

5. G. Gupta and M. Younis. Fault-tolerant clustering of wireless sensor networks. Proceedings of IEEE WCNC. pp. 1579-1584, 2003.

6. G. Gupta and M. Younis. Performance evaluation of load-balanced clustering of wireless sensor networks. 10th Inter. Conf. on Telecomm., vol. 2, pp. 1577-1583, 2003.

7. Y. T. Hou, Y. Shi, J. Pan, and S. F. Midkiff. Lifetime-optimal data routing in wireless sensor networks without flow splitting. In Workshop on Broadband Adv. Sensor Networks, San Jose, CA, 2004.

8. Y. T. Hou, Y. Shi, H. D. Sherali, and S. F. Midkiff. On Energy Provisioning and Relay Node Placement for Wireless Sensor Networks. In IEEE SECON, vol. 32, 2005 .

9. J. Tang, B. Hao, and A. Sen. Relay node placement in large scale wireless sensor networks. Comp. Comm., vol. 29(4), pp. 490-501, 2006.

10. K. Kalpakis, K. Dasgupta, and P. Namjoshi. Maximum Lifetime Data Gathering and Aggregation in Wireless Sensor Networks. IEEE Inter. Conf. on Network., 2002.

11. A. Bari, A. Jaekel and S. Bandyopadhyay. Optimal load balanced clustering in two-tiered sensor networks. In IEEE BaseNets, 2006.

12. E. Falck, P. Floren, P. Kaski, J. Kohonen, and P. Orponen. Balanced data gathering in energy-constrained sensor networks, vol. 3121 LNCS, pp. 59-70, Berlin Heidelberg, 2004. Springer-Verlag.

13. M. Borghini, F. Cuomo, T. Melodia, U. Monaco and F. Ricciato. Optimal data delivery in wireless sensor networks in the energy and latency domains. WICON, pp. 138-145, 2005.

14. M. Yarvis, N. Kushalnagar, H. Singh, A. Rangarajan, Y. Liu nad S. Singh. Exploiting heterogeneity in sensor networks. INFOCOM 2005, vol. 2, pp. 878-890, 2005.

15. ILOG CPLEX 9.1 Documentation. Available at the website http://www.columbia. edu/dano/resources/cplex91_man/index.html 